\section{TECHNICAL NOTE}

\author{
G. Brasil Caseiras \\ J.S. Thornton \\ T. Yousry \\ C. Benton \\ J. Rees
}

A.D. Waldman

H.R. Jäger

\section{Inclusion or Exclusion of Intratumoral Vessels in Relative Cerebral Blood Volume Characterization in Low-Grade Gliomas: Does It Make a Difference?}

SUMMARY: We assessed the influence of inclusion (method 1) and exclusion (method 2) of intratumoral vessels when determining maximum relative cerebral blood volume (rCBVmax) in 3 types of low-grade gliomas (LGGs): astrocytomas, oligoastrocytomas, and oligodendrogliomas. Method 1 yielded significantly higher mean rCBVmax than method 2. However, only method 2 demonstrated a significant $(P=.026$ ) association between $r C B V m a x$ and membership of a differently ranked histologic category. Exclusion of intratumoral vessels appears, therefore, preferable when determining rCBVmax in LGGs.
D ynamic susceptibility contrast-enhanced (DSC) MR perfusion imaging has become an important technique for studying brain tumors. Intratumoral relative cerebral blood volume (CBV $[\mathrm{rCBV}])$ correlates with histologic and angiographic measures of tumor vascularity, ${ }^{1}$ helps predict the histologic grade of gliomas, ${ }^{2}$ and correlates with outcome in lowgrade gliomas (LGGs). ${ }^{3}$ High-grade gliomas usually have a higher rCBV than low-grade tumors, but rCBVs may also be elevated in low-grade oligodendrogliomas (ODs), ${ }^{4}$ which confounds tumor grading. ${ }^{5}$

Techniques of data acquisition, postprocessing, and analysis influence rCBV measurements. Gradient-echo echo-planar imaging (GE-EPI) pulse sequences are more sensitive to larger vessels than spin-echo echo-planar imaging (SE-EPI) methods and provide better differentiation between histopathologic tumor grades. ${ }^{6}$

Wetzel et $\mathrm{al}^{7}$ found that interobserver and intraobserver reproducibility for intratumoral $\mathrm{rCBV}$ were best when the highest CBV from several regions of interest (ROIs) was chosen and highlighted the importance of excluding large vessels. Inclusion or exclusion of intratumoral vessels is often not explicitly stated in glioma perfusion studies, though some investigators focused on excluding peritumoral vessels. ${ }^{6}$ The significance of intratumoral vessels for rCBV measurements has, to our knowledge, not been formally examined.

Here we studied the influence of intratumoral vessels on $\mathrm{rCBV}$ characterization in 3 histologic categories of low-grade glial tumors: astrocytomas (ACs), ODs, and oligoastrocytomas (OAs).

Received June 20, 2007; accepted after revision September 10.

From the Lysholm Department of Neuroradiology (G.B.C., J.S.T., T.Y., H.R.J.), Institute of Neurology (G.B.C., T.Y., C.B., J.R., H.R.J.), University College London, London, United Kingdom; and Department of Imaging (A.D.W.), Charing Cross Hospital, London, United Kingdom.

Please address correspondence to Gisele Brasil Caseiras, Queen Square, Box 65, London, United Kingdom WC1N 3BG; e-mail: g.brasil@ion.ucl.ac.uk

indicates supplemental on-line table.

DOI 10.3174/ajnr.A0993

\section{Technique and Results}

Thirty-four patients with LGGs, composed of $21 \mathrm{ACs}, 8$ ODs, and 5 OAs, had DSC GE-EPI $\left(\mathrm{TR}=1200 \mathrm{~ms}\right.$; TE $=40 \mathrm{~ms}$; flip angle $=20^{\circ}$; FOV $=26 \mathrm{~cm}$; matrix $=96 \times 128$; section thickness $=5 \mathrm{~mm})$ at $1.5 \mathrm{~T}$ (GE Signa Horizon Echospeed LX9.1; GE Healthcare, Waukesha, Wis) with a bolus of $0.1 \mathrm{mmol} / \mathrm{kg}$ of body weight of gadoterate meglumine at $5 \mathrm{~mL} / \mathrm{s}$. Color maps of rCBV were generated with FuncTool 1.9 (GE Healthcare) and analyzed by 2 neuroradiologists (H.R.J. and G.B.C., with 15 and 7 years of experience, respectively), reaching a consensus for placement of ROIs. At least 6 intratumoral ROIs with a size of 9 pixels were placed over areas showing mostly elevated CBV on color perfusion maps. Blood vessels within the tumor were identified on unprocessed perfusion images acquired between the time points of maximum arterial and venous signal intensity drop. Sections above and below intratumoral vessels were viewed to identify potentially confounding large-vessel partial volume effects. We used 2 different methods for selecting the ROI with the maximum intratumoral CBV: method 1 included and method 2 excluded ROIs situated over intratumoral blood vessels and associated partial volume effects. The maximum rCBV (rCBVmax) was then obtained by dividing the highest intratumoral $\mathrm{CBV}$ by the mean $\mathrm{CBV}$ obtained from a contralateral normal-appearing white matter ROI (Fig 1).

Mean rCBVmaxs obtained for each group with each method are shown in the Table. Method 1 yielded higher mean values and wider ranges than method 2 in all 3 of the histologic tumor types, particularly in OA and OD. For the patient group as a whole, there was a significant difference between mean rCBVmax obtained using each method ( $P<.001$, Wilcoxon test). Ordinal regression was used to assess the relationship of rCBVmax and tumor histology, categorized in 3 groups (0: OA; 1 : AC; 2 : OD). Only method 2 showed a significant association between $\mathrm{rCBVmax}$ and the risk of being in a histologic category with a higher ordinate. Using method 2, the odds ratio of being in a higher category was 4.25 (95\% confidence interval: 1.1915.14 for each additional unit rCBV increment; $P=.026$ ). Method 1 did not demonstrate a significant association between rCBVmax and the risk of being in a higher category $(P=.638)$.

\section{Discussion}

Several studies have demonstrated increased rCBV in highgrade gliomas compared with low-grade tumors. ${ }^{2,4-6,8-10}$ 

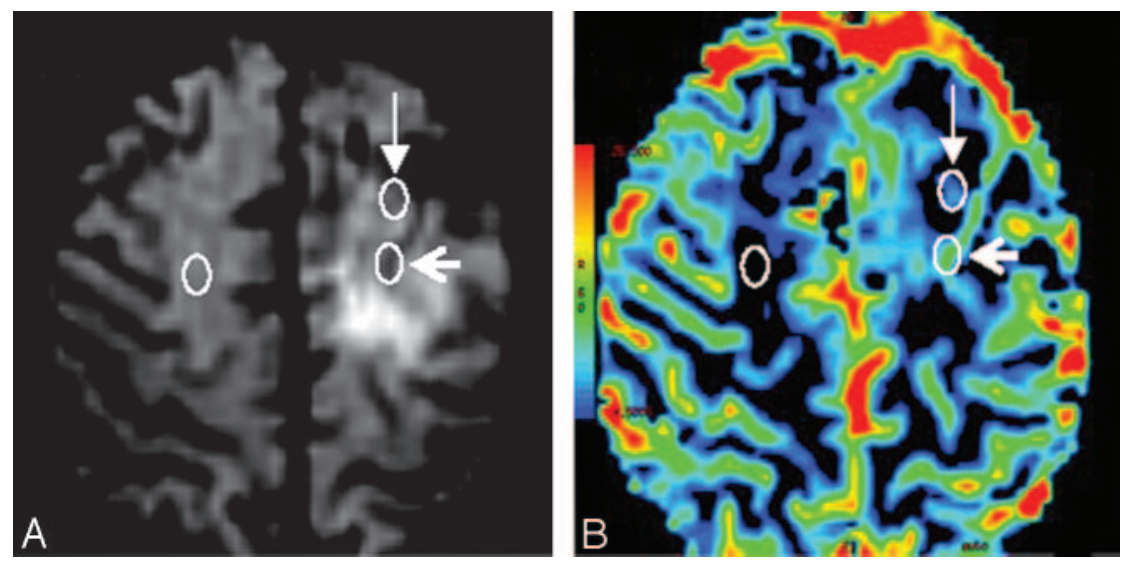

Fig 1. T2*-weighted image during maximum arterial signa intensity drop $(A)$ and rCBV map $(B)$ in low-grade OD, demonstrating the position of ROls used to calculate rCBVmax. Method 1 (open arrowhead, posterior ROI in the left cerebral hemisphere, overlying an intratumoral vessel) and method 2 (closed arrowhead, anterior ROI in the left cerebral hemisphere, lying outside intratumoral vessels). The mean CBV from contralateral normal-appearing white matter (ROI in the right cerebral hemisphere) was used to normalize the data for each method. White circles have been superimposed on the original color-coded ROls generated by FuncTool.

\begin{tabular}{|c|c|c|c|c|}
\hline \multicolumn{5}{|c|}{ rCBVmax obtained using methods 1 and 2} \\
\hline Method & $\begin{array}{c}\text { All Tumors, Mean } \\
\text { (range) }\end{array}$ & $\begin{array}{c}A C(n=21), \text { Mean } \\
\text { (range) }\end{array}$ & $\begin{array}{c}\mathrm{OA}(\mathrm{n}=8), \text { Mean } \\
\text { (range) }\end{array}$ & $\begin{array}{c}\mathrm{OD}(\mathrm{n}=5), \text { Mean } \\
\text { (range) }\end{array}$ \\
\hline Method 1 & $4.01(1.54-6.99)$ & $3.30(1.54-6.65)$ & $5.33(3.07-6.65)$ & $5.04(2.74-6.99)$ \\
\hline Method 2 & $1.63(0.70-3.51)^{*}$ & $1.44(0.70-2.46)$ & $1.53(1.26-1.94)$ & $2.21(1.47-3.51)$ \\
\hline
\end{tabular}

Note:-AC indicates astrocytoma; $\mathrm{OA}$, oligoastrocytoma; $\mathrm{OD}$, oligodendroglioma; rCBV max, maximum relative cerebral blood volume.

* $P<.001$ for method 1 versus method 2 by Wilcoxon test.

There is, however, considerable variation in the reported rCBV values for low- and high-grade tumors (On-line Table). In SE-EPI DSC imaging, transverse relaxation rates peak at a vessel diameter of 1-2 $\mu \mathrm{m}$, whereas in GE-EPI DSC imaging they plateau at 3-4 $\mu \mathrm{m}$ and then remain independent of vessel size, explaining the lower rCBVs found with SE techniques. ${ }^{6}$ Differences may also be due to variations in the histologic types of LGG examined and inclusion or exclusion of intratumoral vessels in the analysis, often not specified.

We demonstrated that inclusion of large intratumoral vessels significantly increases rCBVmax values in all types of LGG. Their identification may be difficult on rCBV color maps alone and necessitates reviewing of unprocessed perfusion data. The diameter of intratumoral vessels clearly identifiable on GE-EPI images lies in the millimeter range (approximating the size of peripheral leptomeningeal vessels), whereas glioma neoangiogenetic vessels in animal models measure between 40 and $250 \mu \mathrm{m}$. $^{6}$

In concordance with previous investigators, we found higher rCBVmax in tumors with oligodendral elements than in purely astrocytic tumors, ${ }^{5}$ explained by the "chicken wire" hypervascularity seen in the former. Cha et $\mathrm{al}^{4}$ chose intratumoral ROIs with an automated method targeting areas of maximum signal intensity decrease during the first pass of the gadolinium-based contrast bolus. This method probably incorporated intratumoral vessels and yielded mean rCBV for ODs of 3.68. Spampinato et $\mathrm{al}^{8}$ presented one of the few reports specifying exclusion of large intratumoral vessels for ROI analysis. Their mean rCBV measurement for a mixed group of low-grade OA and OD (1.61) lies between our group mean rCBV measurements of OA (1.53) and OD (2.21) using method 2. Our findings highlight the importance of using a consistent ROI placement technique, particularly if rCBV data are to be pooled in multicenter studies. The relationship between rCBVmax on MR perfusion imaging and histologic classification as $\mathrm{OA}$ versus $\mathrm{OD}$ has not been reported previously. We were able to demonstrate significant association be- tween maximum intratumoral $\mathrm{rCBV}$ values and histopathologic classification as $\mathrm{AC}, \mathrm{OD}$, and $\mathrm{AO}$, but only when using method 2. As a preferred technique we, therefore, recommend exclusion of intratumoral vessels when determining maximum intratumoral rCBV from GE-EPI DSC-derived data. Although beyond the scope of this work, future research will address the influence of intratumoral vessels on rCBV measurements in high-grade gliomas, which are naturally subject to greater variability.

\section{References}

1. Sugahara T, Koroghi Y, Kochi M, et al. Correlation of MR imaging-determined cerebral blood maps with histologic and angiographic determination of vascularity of gliomas. AJR Am J Roentgenol 1998;171:1479-86

2. Law M, Yang S, Wang $H$, et al. Glioma grading: sensitivity, specificity, and predictive values of perfusion MR imaging and proton MR spectroscopic imaging compared with conventional MR imaging. AJNR Am J Neuroradiol 2003;24:1989-98

3. Law M, Oh S, Babb JS, et al. Low-grade gliomas: dynamic susceptibilityweighted contrast-enhanced perfusion MR imaging-prediction of patient clinical response. Radiology 2006;238:658-67

4. Cha S, Tihan T, Crawford F, et al. Differentiation of low-grade oligodendrogliomas from low-grade astrocytomas by using quantitative blood-volume measurements derived from dynamic susceptibility contrast-enhanced MR imaging. AJNR Am J Neuroradiol 2005;26:266-73

5. Lev MH, Ozsunar Y, Henson JW, et al. Glial tumor grading and outcome prediction using dynamic spin-echo MR susceptibility mapping compared with conventional contrast-enhanced MR: confounding effect of elevated rCBV of oligodendrogliomas [corrected]. AJNR Am J Neuroradiol 2004;25:214-21

6. Sugahara T, Korogi Y, Kochi M, et al. Perfusion-sensitive MR imaging of gliomas: comparison between gradient-echo and spin-echo echo-planar imaging techniques. AJNR Am J Neuroradiol 2001;22:1306-15

7. Wetzel SG, Cha S, Johnson G, et al. Relative cerebral blood volume measurements in intracranial mass lesions: interobserver and intraobserver reproducibility study. Radiology 2002;224:797-803

8. Spampinato MV, Smith JK, Kwock L, et al. Cerebral blood volume measurements and proton MR spectroscopy in grading of oligodendroglial tumors. AJR Am J Roentgenol 2007;188:204-12

9. Yang D, Korogi T, Sugahara T, et al. Cerebral gliomas: prospective comparison of multivoxel 2D chemical-shift imaging proton MR spectroscopy, echoplanar perfusion and diffusion-weighted MRI. Neuroradiol 2002;44:656-66

10. Hakyemez B, Erdogan C, Ercan I, et al. High-grade and low-grade gliomas differentiation by using perfusion MR imaging. Clin Radiol 2005;60:493502 